\title{
Structural Analysis And Molecular Characteristics of SARSCoV-2 Spike Protein Following S494P Point Mutation: Molecular Dynamics Study
}

\author{
Mehr Ali Mahmood Janlou ( $\square$ mehr.janlou@gmail.com ) \\ Gorgan Islamic Azad University \\ Hassan sahebjamee \\ Islamic Azad University Varamin-Pishva Branch \\ Shademan Shokravi \\ Gorgan Islamic Azad University
}

Research Article
Keywords: SARS-CoV-2, Spike protein, RBD, S494P, MD simulation, Docking

Posted Date: July 23rd, 2021

DOl: https://doi.org/10.21203/rs.3.rs-625623/v1

License: (c) (i) This work is licensed under a Creative Commons Attribution 4.0 International License. Read Full License 


\section{Abstract}

The emergence of some mutations in the SARS-CoV-2 receptor binding domain (RBD) can increase the spread and pathogenicity due to the conformational changes and increase the stability of Spike protein. Due to the formation of different strains of SARS-CoV-2 by mutations, and their catastrophic effect on public health, the study of the effect of mutations by scientists and researchers around the world is inevitable. According to available evidence, the S494P variant is observed in several SARS-CoV-2 strains from Michigan, USA. To investigate how the S494P natural mutation alters receptor binding affinity in RBD, we performed structural analysis of wild-type and mutant spike proteins using some bioinformatics and computational tools. The results show that S494P mutation increases the spike protein stability. Also, applying docking by HADDOCK displayed higher binding affinity to hACE2 for mutant spike than wild type possibly due to the increased $\beta$-strand and Turn secondary structures which increases surface accessibly surface area (SASA) and chance of interaction. The analysis of S494P as a critical RBD mutation may provide the continuing surveillance of spike mutations to aid in the development of COVID-19 drugs and vaccines.

\section{Introduction}

SARS-CoV-2 belongs to the subfamily Coronavirinae, a member of the family Coronaviridae, is enveloped, positive-strand RNA viruses and can infect both animals and humans [1].The viruses are subdivided, based on genotypic and serological characters, into four genera: Alpha-, BetaGamma; and Deltacoronavirus [2, 3]. Thus far, all identified CoVs that can infect humans belong to the alphacoronaviruses (alphaCoVs) hCoVNL63 and hCoV-229E and the betacoronaviruses (betaCoVs) HCoV-OC43, HKU1, SARS-CoV, and MERS-CoV [4]. In human, SARS-CoV-2 like SARS and MERS causes respiratory disease called coronavirus disease 2019 (COVID-19) [5]. The first known genus was identified in Wuhan, China, in December 2019 [6, 7]. Since then, many variant have formed and spread around the world, leading to the global pandemic attributed to COVID19 , which is known as one of the dead list pandemics in history. In addition, another consequence of the COVID-19 is the severe recession in the global economy resulting in a dramatic loss of livelihoods and income on a global scale. Under these circumstances, the study and research of scientists and researchers around the world has led to a great deal of knowledge about SARS-CoV-2. This knowledge will greatly help to find and improve vaccines and therapeutics to halt the coronavirus pandemic.

Among several viral proteins, one such major vaccine and antibody target is the spike glycoprotein (S-protein) of the coronavirus which has an essential role in virus entry into the host cell and pathogenesis of SARS-CoV-2 [8, 9]. The length of the spike protein is about $20 \mathrm{~nm}$ and it is a homotrimer protein whit two functional subunits S1 and S2 per monomer. The S1 subunit forms the spike head and recognizes human angiotensin-converting enzyme 2 (hACE2) [10]. The receptor-binding domain (RBD) which recognizes the host cell surface receptor (hACE2) and is responsible for virus attachment at the cell surface, is located within $S 1[11,12]$. The $S 2$ subunit forms a stem that anchors the spike in the viral envelope and fused the viral particles to the cell membrane. RBD has a region called the receptor binding motif (RBM). RBM directly binds to the viral binding motif (VBM) in hACE2 [13]. The important structural features of the spike are illustrated in Fig. 1. Spike variants that have a greater virus-receptor interaction are easily spread and become infections [12]. Given what may be an exponential increase in infections, there is expected to be a higher rate of morbidity or mortality. According to available evidence, the S494P variant is observed in several SARS-CoV-2 strains from Michigan, USA [14]. However, no detailed study has been done on structural changes of spike protein due to S494P mutation and binding affinity for hACE2. Here, for a better understanding, we investigated the structural and molecular properties of S494P mutation using of bioinformatics tools and molecular dynamics simulation.

\section{Methods}

\subsection{Stability investigation}

There is a close relationship between stability and structural energy of proteins. It is also determine the stability changes caused by the amino acid substitution of proteins [15]. Hence, one of the most common approaches to trace the effect of mutations on protein stability is structural energy calculation. To date, numerous computational tools have been developed to predict the effect of mutations on stability. However, only a few of these tools have been employed to improve protein stability experimentally through the use of point mutation [16]. So, we selected two popular tools means FoldX (version 5) plugin for YASARA [17] and PoPMuSiCv3.1 [18] to calculate and compare the energy of the structure in the wild and mutant spike. FoldX calculate the energy of the structure by applying linear combination of physics and statistics-based energy terms algorithms [19]. It provides useful information about the characteristics of protein conformations by calculating the different terms of energy.

\subsection{Molecular Dynamics evaluation}

Molecular dynamics simulation is a high speed, and flexibility of approach carried out to find out the behavior and movement of atoms and molecules over a given period of time [20]. The 3D structure of spike with PDB ID: 6M0J was retrieved from the protein databank (http:// www.rcsb.org/pdb)[21]. Simulations for wild-type (WT) and mutant spike proteins were performed using the GROMACS package (version 2019.1) with Amber03 force field [22-24] and run for $100 \mathrm{~nm}$. The 6MOJ X-ray structure was placed in a cubic box containing approximately 
7285 SPC 216 water molecules and using periodic boundary condition. The distance from the cubic-box edges was set to $1 \mathrm{~nm}$. In order to neutralize the net charge of systems $2 \mathrm{Cl}^{-}$ions were added. Periodic boundary conditions (PBC) were exerted in all three directions of the system. Then, the entire system was minimized using the steepest descent of 1000 steps followed by the conjugate gradients of 10000 steps [25]. In the equilibration step an NVT ensemble with a constant temperature of $300 \mathrm{~K}$ for $200 \mathrm{ps}$, and NPT ensemble with a constant pressure of 1 bar for 500 ps were applied. Berendsen [26] and Parrinello-Rahman algorithms [27] were applied to keep the pressure and temperature constant at 1 bar and 300 Position restraint process was used in NVT and NPT ensemble steps. We performed two independent MD runs, from wiled and mutant structures in order to ensure that results are significant for the relevant mutation. We then computed the comparative analysis of structural deviations in wiled and mutant structures. Root-mean-square-deviation (RMSD), Root- mean-square-fluctuation (RMSF), and surface accessibly surface area (SASA) were measured for target structures..

\subsection{Principal component analysis (PCA) and Free energy landscape (FEL)}

It is used by principle component analysis (PCA) as a standard tool in statistical mechanics to determine the correlated motions of the residues to a set of linearly uncorrelated variables called principle component. This method was carried out to construction the linear transformation of characteristics of the covariance matrix and reduction of the instantaneous linear correlations among the coordinates of the simulated proteins. In this study, Ca atoms of target proteins were selected to estimation of fluctuations, provides significant characteristics to essential internal motions. Results obtained from PCA were subjected to free energy landscape assessment (FEL), to describe the probability energy distribution of one or more collective variable of the protein system, which helps to visualize the stability of different conformations for a protein [28]. Extraction of 2D representations of the FEL from the trajectory was carried out using g_sham in GROMACS [29].

\subsection{Immunogenicity characteristics of spike Protein}

Immunogenicity is a humeral or cellular immune response in the body of a human administered a drug recognizes it as foreign. Cytotoxic CD $8+$ T lymphocytes (CTLs) directly kill virus-infected cells in the respiratory tract. Identification of epitopes of viral antigens that can be effectively delivered to $\mathrm{T}$ lymphocytes to elicit a protective response for the host is important in vaccine development [30]. At the same time, antigensupplying cells (APCs) play a major role in the presentation of antigenic peptides by human leukocyte (HLA) class I molecule to CD8 + lymphocytes [31]. HLA molecules with very high polymorphisms can present viral antigen peptides to $T$ lymphocytes, causing a variety of immune responses in different individuals [32]. Due to structural changes of point mutation proteins, it is expected that the immunogenicity of the mutant spike proteins could also be changed. In this study, the IEDB [33] server was used to evaluate and compare the immunogenicity of the wild type and mutant spikes. T-cell epitope prediction was accomplished by MHC I binding prediction options.

\subsection{Docking}

To investigate the effect of the S494P mutation on the ability of spike to bind to the hACE2 receptor, docking process was utilized. HADDOCK is a flexible docking program where experimental or bioinformatics information is directly used to predicting the binding affinity of protein-protein interactions [34]. It is user-friendly software, which uses biochemical or biophysical information to enhance sampling and scoring [34]. We have implemented some restrictions to run this software, the active and passive residues were our restrictions. According to [35], and analysis of some existing crystal structures with PDB ID 6VW1, 6LZG, and 6M17 active residues were identified (Table 1). During docking, the solvated docking mode was activated and active residues were considered as the fully-flexible segment. Passive residues that were close to the active residues were chosen automatically. The cluster with the best score was used during the docking for analysis. The LigPlot v.2.2 was applied to determine schematic diagrams of the protein-protein interactions [36].

Table 1

Name and position of the SARS-CoV-2 and hACE2 contact residues.

\begin{tabular}{|ll|}
\hline SARS- CoV-2 & K417, G446, Y449, Y453, L455, F456, A475, F486, N487, Y489, Q493, G496, Q498, T500, N501, G502, Y505 \\
\hline hACE2 & Ser19, Q24, F28, D30, K31, H34, E35, E37, D38, Y41, Q42, L79, M82, Y83, N330, K353, G354, D355, R357, R393
\end{tabular}

\section{Results And Discussion}

\subsection{Stability investigation}

The most important forces for protein stability are intramolecular interactions, i.e. disulfide bridges, ion interactions, hydrogen bonds, hydrophobic interactions and core packing [37]. The core function of FoldX and PoPMuSiC, the empirical force field algorithm, is based on free energy $(\Delta \mathrm{G})$ terms aiming to calculate the change of $\Delta \mathrm{G}$ in $\mathrm{kcalmol}^{-1}$ (Eq. (1)).

$\Delta \mathrm{G}=\mathrm{a} \Delta \mathrm{Gvdw}+\mathrm{b} \Delta \mathrm{GsolvH}+\mathrm{c} \Delta \mathrm{GsolvP}+\mathrm{d} \Delta \mathrm{Gwb}+\mathrm{e} \Delta$ Ghbond $+\mathrm{f} \Delta \mathrm{Gel}+\mathrm{g} \Delta \mathrm{Gkon}+\mathrm{h} \mathrm{T} \Delta \mathrm{Smc}+\mathrm{k} \mathrm{T} \Delta \mathrm{Smc}+\mathrm{l} \Delta \mathrm{Gclash}$. (1) 
In this study, both PoPMuSiC and FoldX outputs showed that the P494 increases the stability of spike protein conformation. The $\Delta \Delta \mathrm{G}$ calculated by PoPMuSiC for S494P mutation is $-0.02 \mathrm{kcalmol}^{-1}$.( $\Delta \Delta \mathrm{G}<0$ means stabilizing mutation). The total energy calculated by FoldX was 60.62 and $56.41 \mathrm{kcalmol}^{-1}$ for wild type, and S494P spike protein, respectively. According to Table 2, substitution of S494 by P decreases SideHbond from -41.40 to $-50.56 \mathrm{kcalmol}^{-1}$. The lower energy content of S494P mutation indicates that P367 mutation increases the stability of spike protein. So, it can impact the performance of the SARS-CoV-2.

Table 2 Energy parameters measured by FoldX for the native and mutant spike. (the unit of all energy terms is $\mathrm{kcalmol}^{-1}$ ).

\begin{tabular}{|llllllllll|}
\hline FoldX & BackHbond & SideHbond & Energy_VdW & Electrostatic & $\begin{array}{l}\text { Energy } \\
\text { SolvP }\end{array}$ & $\begin{array}{l}\text { Energy } \\
\text { _SolvH }\end{array}$ & $\begin{array}{l}\text { Energy_ } \\
\text { vdwc } \\
\text { lash }\end{array}$ & $\begin{array}{l}\text { Energy_ Entropy_sidec } \\
\text { torsion }\end{array}$ & $\begin{array}{l}\text { Entropy_ } \\
\text { mainc }\end{array}$ \\
\hline S494 & -101.05 & -41.40 & -220.30 & -14.66 & 309.78 & -278.13 & 16.16 & 6.42 & 112.23 \\
\hline P494 & -101.04 & -50.56 & -220.55 & -14.68 & 308.43 & -279.61 & 16.08 & 6.45 & 112.16 \\
\hline
\end{tabular}

\subsection{Molecular dynamics simulation analysis}

We next performed 100 ns MD simulation to analyze carefully the structural consequences of mutating residue S494P, which is naturally occurred in spike protein.

The stability of the protein relative to its conformation can be determined by the deviations produced during the simulation trajectory. The RMSD value is used for measuring the difference between the backbones of a protein from its initial structural conformation to its final position. The smaller the deviations, the more stable the protein structure. From the RMSD simulation shown in Fig. 2.A, it can be observed that the systems equilibrated after $40 \mathrm{~ns}$. Also, the structure of the mutant shows a significant deviation from wild type. The average value of fluctuations in backbone RMSD during the last $60 \mathrm{~ns}$ is depicted in Table 3. The lower RMSD values for mutant structure indicate that the overall topology has changed so that S494P shows a stable structure than wild type system.

The residue-based root mean square fluctuation (RMSF) of the backbone for the S494P displayed whit less flexibility in the residue number 415-485, which is a receptor binding motif (RBM) region of spike protein compared to the wild type. This indicates that the secondary structure of RBD was stable. Accordingly, the RMSF results also confirm that S494P system is more stable than wild type (Fig. 2.B).

Table 3 The average value of RMSD, and RMSF during the simulation for wild type and mutant structures.

\begin{tabular}{|lll|}
\hline Parameters & Wild type & S494P \\
\hline RMSD $^{1}$ & $0.494 \pm 0.078(\mathrm{~nm})$ & $0.410 \pm 0.049(\mathrm{~nm})$ \\
RMSF $^{2}$ & $0.163 \pm 0.12(\mathrm{~nm})$ & $0.147 \pm 0.14(\mathrm{~nm})$ \\
\hline SASA & $108.40 \pm 2.03$ & $110.9 \pm 2.47(\mathrm{~nm})$ \\
\hline 1The average value during the last $60 \mathrm{~ns}$ \\
\hline
\end{tabular}

Another important structural parameter, that provides important information about the structural plasticity of proteins, is the time-dependent secondary structures which calculated using DSSP classification of secondary structure elements. Analysis of DSSP results shows that in the mutant structure the tendency to forming of $\beta$-sheet, bend and turn secondary structures have increased but coil and a-helix reduced (Fig. 3). In general, it can be said that the S494P mutation cause an increase the sum of the regular structures. Since the tow bend and turn secondary structures tend to be at the protein surface, it can increase the available surface area of the mutant spike and the chance of the interaction of mutant spike with wild type receptor protein. Determining the amount of SASA also confirm this claim (Fig. 3).

\subsection{Free energy landscape (FEL)}

To indicate structural properties using thermodynamic information, we constructed a free energy landscape (FEL) for wiled type and mutant spike proteins (Fig. 4). On the FEL, the energy minima was obtained based on the probability of a combination of MD data points to map the minimum energy configuration of the proteins. As seen in Fig. 4, the red and yellow colors indicate lowest energy based on the stability of the spike protein. It can be said that according to the probability energy minima distribution, stability of S494P is higher than wild type.

\subsection{Immunogenicity evaluation}


At this stage, the immunogenicity of S494P mutation relative to wild type was obtained using MHC I binding prediction. Evaluation of the immunogenicity of mutant spike proteins Indicates that they have different immunogenic responses. Based on the results, it can be said that P494 has a lower immunogenic capacity than the wild type (Table 4). Given that, increased stability can decrease the formation of aggregates and consequently, immunogenicity [38-40]; this may be why the immunogenicity of P494 is reduced.

Table 4

Average score reported for T-cell epitopes. The selected length for MHC-I was 9.

\begin{tabular}{|ll|}
\hline sequence & MHC-I \\
\hline S494 & 0.011 \\
\hline P494 & 0.005 \\
\hline
\end{tabular}

\subsection{Docking analysis}

After running HADDOCK, the best cluster was selected according to the size of the cluster and its Z -score. To find out the affinity level of biological partner, total binding energy which is the sum of Van der Waals and Electrostatic energy is computed [41]. According to the results of ducking analysis, the total binding energy of wild type and P494 spikes with their receptors were -339.8 and $-348.1 \mathrm{kcalmol}^{-1}$, respectively. Also, the Buried Surface Area (BSA), which measures the size of the interface in a protein-protein complex, in the spike ${ }^{\mathrm{P} 494}$ with its receptor is more than wild type complex (Table 5). The increase in BSA is due to a conformational change of spike ${ }^{\mathrm{P} 494}$ compared to the wild type and it can increase the chance of the interaction. Then HADDOCK results show better binding of spike ${ }^{\mathrm{P} 494}$ to its receptor relative to wild type complex.

For more details, changes in the residue binding patterns of wild type and mutant spike complexes were investigated by LigPlot (Fig. 5). Valuable information has been obtained about the spike-hACE2 molecular interactions. The type and number of some of the interface amino acids interactions are different. The number of Hbound in the wild type and spike ${ }^{\mathrm{P} 494}$ complexes are 15 , and 16 respectively. Also, in the wild type, for example, the Ala475 forms one Hbound with Ser19 of hACE2, but do not have any role in interaction of mutant whit hACE2. In addition, the Thr500 form two Hbounds with Tyr41 in the wild spike. However, on the spike ${ }^{\mathrm{P} 494} \mathrm{Thr} 500$ forms one Hbound with Tyr41 and the other with Arg357. Moreover, Lys353 of hACE2 in complexes with spike ${ }^{P 494}$ forms three Hbounds with Gln498, Gly496 and Asp501 residues, while in the interaction between wild spike, it forms two Hbounds with GIn498 and Gly496. Table 6 details the comparison of Hbounds between amino acids of spike-hACE2 complexes.

The effect of mutations on the binding pattern could be the result of structural changes in spike proteins through a new substitution. Figure 6 shows the structural changes related to area neighbor of mutant positions in the spike proteins. Comparison of physicochemical properties of wild and mutant residue showed that in the S494P mutation, Ser is the simplest hydroxyl amino acid and low side chain flexibility. On the other hand, free Pro is a secondary amine with the side chain curving back from the alpha carbon to bond to the amine nitrogen. When incorporated into a protein, Pro therefore lacks an amide proton and locks the phi backbone angle approximately $-60^{\circ}$ by the pyrrolidine ring and psi angles fall into two groupings near $-45^{\circ}$ and $+135^{\circ}$ in the helical and sheet structures. The backbone near a pro thus tends to be inflexible and may prevent certain secondary structures from forming. 
Table 5

The statistics of the best clusters for native and mutant spike in interaction with hACE2.

\begin{tabular}{|lll|}
\hline Haddock criteria & Wiled type & S494P \\
\hline HADDOCK score & $-154.3+/-3.9$ & $-157.7+/-1.1$ \\
\hline Cluster size & 199 & 198 \\
\hline RMSD from the overall lowest-energy structure & $0.7+/-0.5$ & $0.7+/-0.4$ \\
\hline Van der Waals energy & $-68.8+/-4.0$ & $-68.3+/-3.4$ \\
\hline Electrostatic energy & $-261.1+/-15.8$ & $-272.2+/-11.8$ \\
\hline Desolvation energy & $-36.3+/-2.8$ & $-32.0+/-2.7$ \\
\hline Restrains violation energy & $0.0+/-0.03$ & $0.1+/-0.11$ \\
\hline Buried Surface Area $\left(\AA^{2}\right)$ & $1950.4+/-93.3$ & $1976.0+/-68.9$ \\
\hline Z-Score & 0.0 & 0.0 \\
\hline Total Interaction energy & -339.8 & -348.1 \\
\hline
\end{tabular}

Table 6

Comparison of hydrogen bound between amino acids of spike-hACE2 complexes.

\begin{tabular}{|lll|}
\hline S 1 hACE2 & S $_{1}$ hACE2 & S $_{1}$ hACE2 \\
\hline Ala475 (WT) Ser19 & Tyr449 (WT) Asp38 & Gly446 (WT) Gln42 \\
Ala475 (S494P) - & Tyr449 (S494P) Asp38 & Gly446 (S494P) Gln42 \\
\hline Lys417 (WT) Asp30 & Tyr505 (WT) Asp38 & Gly496 (WT) Lys353 \\
Lys417 (S494P) Asp30 & Tyr505 (S494P) - & Gly496 (S494P) Lys353 \\
\hline Gln493 (WT) Lys31 & Gly496 (WT) Asp38 & Gly496 (WT) Asp38 \\
GIn493 (S494P) Lys31 & Gly496 (S494P) - & Gly496 (S494P) - \\
\hline Gln493 (WT) Glu35 & Asn487 (WT) Tyr83 & Tyr449 (WT) GIn42 \\
GIn493 (S494P) Glu35 & Asn487 (S494P) Tyr83 & Tyr449 (S494P) GIn42 \\
\hline Thr500 ${ }^{\text {a }(W T) ~ T y r 41 ~}$ & Asn487 (WT) GIn24 & Lys353 (WT) - \\
Thr500 ${ }^{b}$ (S494P) Tyr41 & Asn487 (S494P) Gln24 & Lys353 (S494P) Gly502 \\
\hline Gln498 (WT) Lys353 & Tyr505 (WT) - & Asp501 (WT) - \\
Gln498 (S494P) Lys353 & Tyr505 (S494P) Glu37 & Asp501 (S494P) Lys353 \\
\hline Arg357 (WT) - & & \\
Arg357 (S494P) Thr500 & & \\
\hline a two hydrogen bound, bone hydrogen bound. & \\
\hline
\end{tabular}

\section{Conclusion}

Although the new SARS-Cov-2 virus (CovID-19) has emerged since 2019, due to global pandemic, many studies are being done on this virus by researchers at the molecular level. In this study, all analyses were appropriate and convergent. Results from some of bioinformatics tools such as MD simulation, FoldX, PoPMuSiC and docking analysis showed increased stability and better interaction of mutant spike with its receptor. It means that S494P mutation results in structural changes in the mutant relative to the wild type structure that can increase binding affinity. Due to the fact that the P494 mutation in close contact whit the hACE2 binding region, the enhanced binding affinity of P494 can be attributed to some factors such as conformational changes and then interfacial complementary during hACE2 recognition and also the reorientation of some of residues at the interface Which makes the number of hydrogen bonds more and stronger. Altogether, further S494P infection may be related to these conformational changes and new structural properties. Therefore, the use of other methods such as experimental stability analysis and S494P immunoassay studies to closely monitor about function, features, and other aspects of this SARS-COVID-19 mutation is important to find specific treatment in the next future. 


\section{Declarations}

\section{Acknowledgement}

We are grateful to Dr hamidreza alaie (Department of physics, Islamic Azad University Varamin , Tehran, Iran) for providing us technical supports.

Corresponding author: Mehr Ali Mahmood Janlou

Email: mehr.janlou@gmail.com

Phone: +989113517834

Fax:+9817-32151913

Funding: This study was (partially or fully) supported by research office of Gorgan Islamic Azad university.

Conflicts of interest/Competing interests: The authors have declared that there is no competing /conflict of interest.

Availability of data and material: Most data generated or analyzed during this study are included in this article. All data will be available upon request of reviewer or journal editorial board.

Code availability: N/A

Authors' contributions:

Mehr Ali Mahmood Janlou: Conception and design, Methodology, Data analysis and interpretation Shadman shokravi: Conception and design , Writing-review and editing hassan sahebjamee: Methodology, Visualization, Collection and assembly of data

All authors read and approved the final manuscript.

\section{References}

1. Gorbalenya A, et al (2020) The species Severe acute respiratory syndrome related coronavirus:classifying 2019-nCoVand naming it SARSCoV-2,Nature Microbiology 5 (4), pp. 536-544. https://doi.org/10.1038/s41564-020-0695-z

2. Lefkowitz EJ, Dempsey DM, Hendrickson RC, Orton RJ, Siddell SG and Smith DB (2018) Virus taxonomy: the database of the InternationalCommittee on Taxonomy of Viruses (ICTV), Nucleic Acids Research, 46, pp. D708- D717. https://doi.org/10.1093/nar/gkx932

3. Adams MJ, and Carstens, E.B (2012) Ratification vote on taxonomic proposals to the International Committee on Taxonomy of Viruses. Arch.Virol, 157, pp. 1411-1422. https://doi.org/10.1007/s00705-012-1299-6

4. Zaki A M, et al (2012) Isolation of a novel coronavirus from a man with pneumonia in Saudi Arabia, N. Engl. J. Med, 367, pp. 1814-1820. https://doi:10.1056/NEJMoa1211721

5. Stanislaw PS, et al (2020) The 2019-2020 Novel Coronavirus (Severe Acute Respiratory Syndrome Coronavirus 2) Pandemic: A Joint American College of Academic International Medicine-World Academic Council of Emergency Medicine Multidisciplinary COVID-19 Working Group Consensus Paper. J Glob Infect Dis, 12(2), pp. 47- 93. http://doi: 10.4103/jgid.jgid_86_20

6. Cristea A E (2020) Histopathological findings in the advanced natural evolution of the SARS-CoV-2 infection, Rom J Morphol Embryol, 61(1), pp 209-218. https://doi.org/10.47162/RJME.61.1.23

7. Ozono S, et al (2021) SARS-CoV-2 D614G spike mutation increases entry efficiency with enhanced ACE2-binding affinity, NATURE COMMUNICATION, 12, pp. 848. https://doi.org/10.1038/s41467-021-21118-2

8. Chan JFW, Kok KH, Zhu Z, Chu H, Wang KK, Yuan To-S, Yuen KY (2020) Genomic characterization of the 2019 novel human-pathogenic coronavirus isolated from a patient with atypical pneumonia after visiting Wuhan, Emerg Microbes Infect, 9, pp. 221-236.

https://doi.org/10.1080/22221751.2020.1719902 
9. Astuti I, Ysrafil (2020) severe acute respiratory syndrome coronavirus 2 (SARS-CoV- 2): an overview of viral structure and host response, Diabetes Metab Syndr Clin Res Rev, 14, pp. 407-412. https://doi.org/10.1016/j.dsx.2020.04.020

10. Devaux CA, Rolain JM, Raoult D (2020) ACE2 receptor polymorphism: susceptibility to SARS-CoV-2, hypertension, multi-organ failure, and COVID-19 disease outcome. J. Microbiol. Immunol. Infect, 53, pp. 425-435. https://doi.org/10.1016/j.jmii.2020.04.015

11. Yan R, Zhang Y, Li Y, Xia L, Guo Y, Zhou Q (2020) Structural basis for the recognition of SARS-CoV-2 by full-length human ACE2. Science, 80, pp 1444-1448. https://doi.org/10.1126/science.abb2762

12. Walls AC, Park YJ, Tortorici MA, Wall A, McGuire AT, Veesler D (2020) Structure, function, and antigenicity of the SARS-CoV-2spike glycoprotein. Cell, 181, pp. 281-292. https://doi.org/10.1016/j.cell.2020.02.058

13. Lan J, Ge J,Yu J, Shan S, Zhou H, Fan S, Zhang Q, Shi X, Wang Q, Zhang L \& Wang X (2020) Structure of the SARS-CoV-2 spike receptorbinding domain bound to the ACE2 receptor. Nature, 581, pp. 215-220. https://doi.org/10.1038/s41586-020-2180-5

14. Chakraborty s (2021) Evolutionary and structural analysis elucidates mutations on SARSCoV2 spike protein with altered human ACE2 binding affinity, Biochemical and Biophysical Research Communications, 534, pp. 374-380. https://doi.org/10.1016/j.bbrc.2020.11.075

15. Funahashi J, Takano K, and YutaniK (2001)Are the parameters of various stabilization factors estimated from mutant human lysozymes compatible with other proteins? Protein Engineering, Design and Selection, 14(2), pp. 127-134. https://doi.org/10.1093/protein/14.2.127

16. Broom A, Jacobi Z, Trainor K, Elizabeth M (2017) Computational tools help improve protein stability but with a solubility tradeoff, J Biol Chem, 292(35), pp.14349-14361. htpps://doi.org/10.1074/jbc.M117.784165

17. Buß O, Rudat J, \& Ochsenreither K (2018) FoldX as protein engineering tool: better than random based approaches?, Computational and Structural Biotechnology Journal, 16, pp. 25-33. https://doi.org/10.1016/j.csbj.2018.01.002

18. Dehouck Y, Grosfils A, Folch B, Gilis D, Bogaerts P, Rooman M (2009) Fast and accurate predictions of protein stability changes upon mutations using statistical potentials and neural networks: Popmusic-2.0, Bioinformatics, 25(19), pp. 2537- 2543.

https://doi.org/10.1093/bioinformatics/btp445

19. Gerasimavicius, Liu X, \& Marsh J A (2020) Identification of pathogenic missense mutations using protein stability predictors, Scientific Reports,10(1), pp. 15387. https://doi.org/10.1038/s41598-020-72404-w

20. Hildebrand PW, Rose A S, \& Tiemann J K S (2019) Bringing molecular dynamics simulation data into view, Trends in Biochemical Sciences, 44(11), 902-913. https://doi.org/10.1016/j.tibs.2019.06.004

21. Zardecki C, Dutta S, David S G, Voigt M, Stephen K B (2016) RCSB Protein Data Bank: A Resource for Chemical, Biochemical, and Structural Explorations of Large and Small Biomolecules, Journal of Chemical Education, 93(3),pp. 569- 575.

https://doi.org/10.1021/acs.jchemed.5b00404

22. Kutzner C, Pall S, Fechner M, Esztermann A, de Groot B L, \& Grubm€uller H (2019) More bang for your buck: Improved use of GPU nodes for GROMACS 2018, Journal of Computational Chemistry, 40(27), pp. 2418-2431. https://doi.org/10.1002/jcc.26011

23. Berendsen HJC, van der Spoel D, van Drunen R (1995) GROMACS: A message-passing parallel molecular dynamics implementation, Comput Phys, 91, pp. 43-56. https://doi.org/10.1016/0010-4655(95)00042-E

24. Zhang D, Lazim R, \& Yip YM (2019) Incorporating polarizability of backbone hydrogen bonds improved folding of short a-helical peptides, Biophysical Journal 117(11), pp. 2079-2086. https://doi.org/10.1016/j.bpj.2019.10.020

25. Li Z, Li S, Wei X, \& Zhao Q (2019) Scaled Alternating Steepest Descent Algorithm Applied for Protein Structure Determination from Nuclear Magnetic Resonance Data, J Comput Biol, 26(9),pp. 1020-1029. https://doi.org/10.1089/cmb.2019.0013

26. Margraf JT, Hennemann M, \& Clark T (2020) EMPIRE: A highly parallel semiempirical molecular orbital program: 3: Born-Oppenheimer molecular dynamics, Journal of Molecular Modeling, 26(3), pp. 43. https://doi.org/10.1007/s00894-020-4293-z

27. Miller RE, Tadmor EB, Gibson JS, Bernstein N, \& Pavia F (2016) Molecular dynamics at constant Cauchy stress, J Chem Phys, 144(18) pp. 184107. https://doi.org/10.1063/1.4948711

28. Aier I, Varadwaj PK, \& Raj U (2016) Structural insights into conformational stability of both wild-type and mutant EZH2 receptor, Sci Rep, 6, pp. 34984. Scientific Reports. https://doi.org/10.1038/srep34984

Page 8/14 
29. Hess B, Kutzner C, van der Spoel D, Lindahl E (2008) GROMACS 4: algorithms for highly efficient, loadbalanced, and scalable molecular simulation, J. Chem. Theory Comput, 4(3), pp435-447. https://doi.org/10.1021/ct700301q

30. Seder RA, Darrah PA, Roederer M (2008) T-cell quality in memory and protection: implications for vaccine design. Nature Reviews Immunology, 8(4), pp. 247-58. https://doi.org/10.1038/nri2274

31. Kumar S, Nyodu R, Maurya VK, Saxena SK (2020) Host immune response and immunobiology of human SARS-CoV-2 infection, Coronavirus Disease 2019 (COVID-19), pp. 43-53. https://doi.org/10.1007/978-981-15-4814-7_5

32. Tavasolian F, Rashidi M, Hatam GR, Jeddi M, Zavaran Hosseini A, Mosawi SH, Abdollahi E and Inman RD (2021) HLA, Immune Response, and Susceptibility to COVID-19, Front. Immunol, 11, pp.3581. https://doi.org/10.3389/fimmu.2020.601886

33. Dhanda SK, Mahajan S, Paul S, Yan Z, Kim H, Jespersen MC, Jurtz V, Andreatta M, Greenbaum J A, Marcatili P, Sette A, Nielsen M, \& Peters B (2019) IEDB-AR: Immune epitope databaseanalysis resource in 2019, Nucleic Acids Res, 47(W1), pp. W502-w506.

https://doi.org/10.1093/nar/gkz452

34. Rolf B, Bonvin J, Alexandre M J, Cyril D (2016) HADDOCK: A Protein-Protein Docking Approach Based on Biochemical or Biophysical Information. J. Am. Chem. Soc, 125, pp. 1731-1737. https://doi.org/10.1021/ja026939x

35. Gomari MM , Rostami N, Hossein Omidi AH, \& Arab SS (2021) Insight into molecular characteristics of SARSCoV- 2 spike protein following D614G point utation, a molecular dynamics stud. Journal of Biomolecular Structure and Dynamics, pp. 1-9.

36. Laskowski, R. A., \& Swindells, M. B (2011) LigPlot: Multiple ligand-protein interaction diagrams for drug discovery, Journal of Chemical Information and Modeling, 51(10), pp. 2778-2786. https://doi.org/10.1021/ ci200227u

37. Vieille C, Zeikus GZ (2001) Hyperthermophilic enzymes: sources, uses, and molecularmechanisms for thermostability, Microbiol Mol Biol Rev, 65, pp.1- 43. https://doi.org/10.1128/MMBR.65.1.1-43.2001

38. Manning MC, Chou DK, Murphy BM, Payne RW, and Katayama DS (2010) Stability of protein pharmaceuticals: an update, Pharm. Res, 27(4) pp. 544-575. https://doi.org/10.1007/s11095-009-0045-6

39. Boulet AM, Byrne B, and Kazarian SG (2014) High-throughput thermal stability analysis of a monoclonal antibody by attenuated total reflection ft-ir spectroscopic imaging, Anal. Chem, 86(19): 9786-9793. https://doi.org/10.1021/ac502529q

40. Redington JM, Breydo L, and Uversky VN (2017) When good goes awry: the aggregation of protein therapeutics, Protein Pept. Lett, 24(4), pp. 340 -347. https://doi.org/10.2174/0929866524666170209153421

41. Kumar A, Purohit R (2014) Use of long term molecular dynamics simulation in predicting cancer associated SNPs, PLoS computational biology, 10(4) pp.e1003318. https://doi.org/10.1371/journal.pcbi.1003318

\section{Figures}




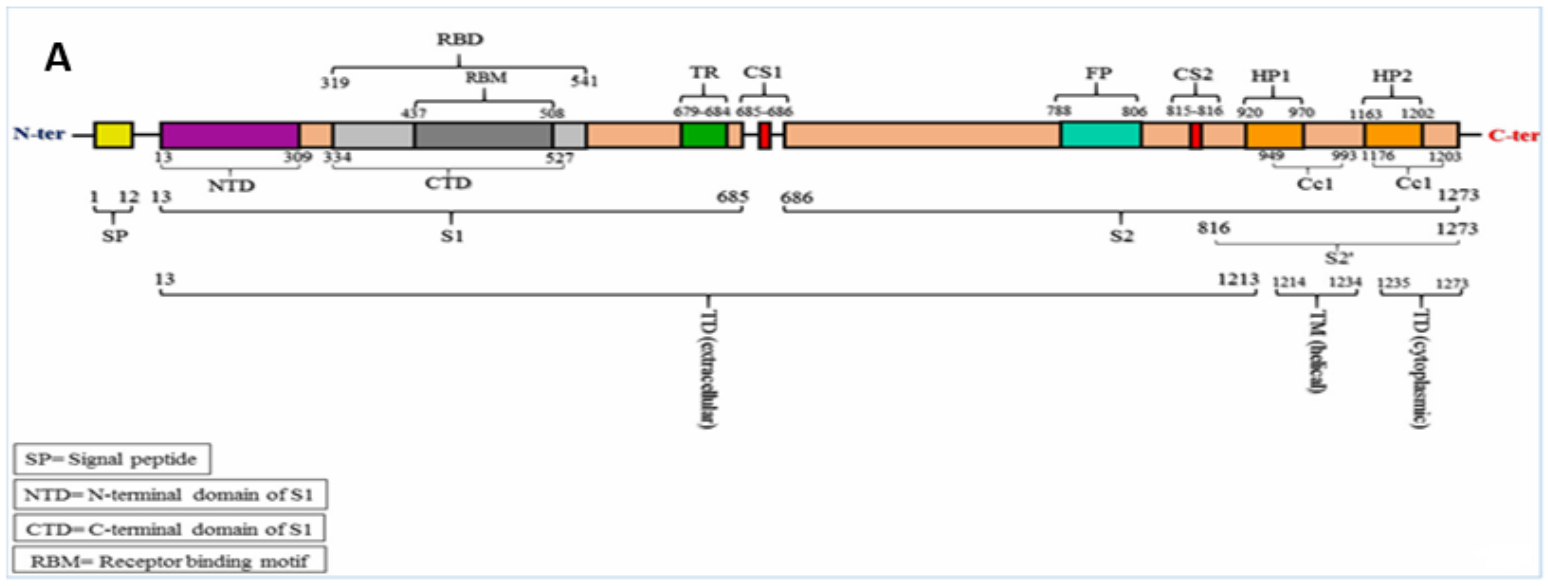

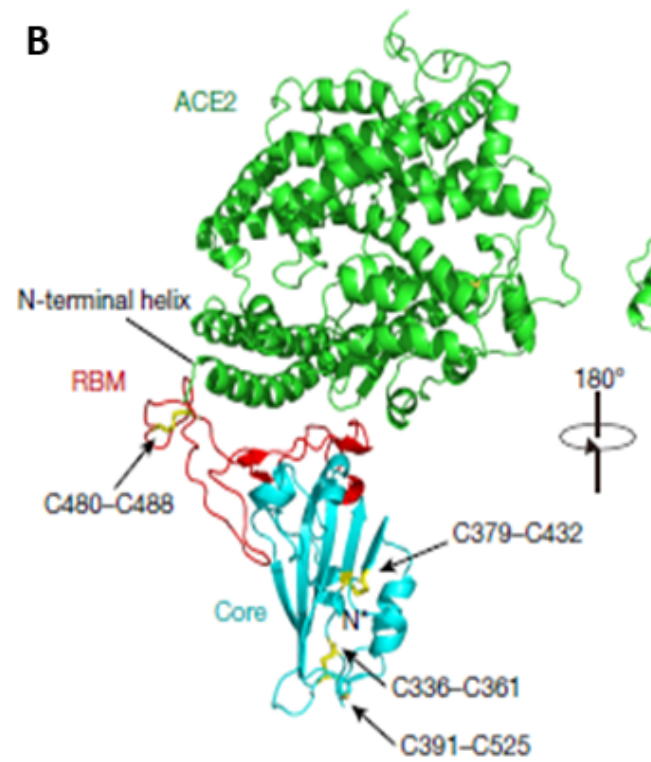

SARS-CoV-2 RBD

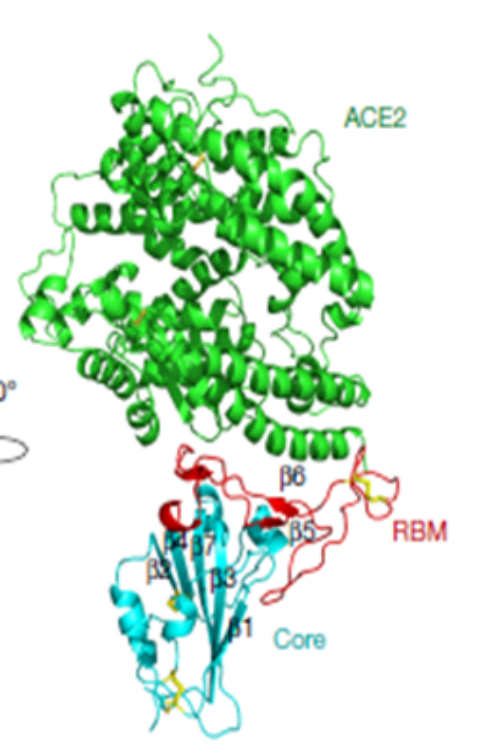

SARS-CoV-2 RBD

\section{Figure 1}

A, Overall topology of the SARS-CoV-2 spike monomer. TD, topological domain; TM, transmembrane domain; TR, Tropism region; HP, heptad repeats; FP, fusion peptide; Cc, coiled coils; NTD, N-terminal domain. B,Overall structure of the SARS-CoV-2 RBD bound to ACE2. ACE2 is shown in green. The SARS-CoV-2 RBD core is shown in cyan and RBM in red. Disulfide bonds in the SARS-CoV-2 RBD are shown as sticks and indicated by arrows. The N-terminal helix of ACE2 responsible for binding is labeled. 
A

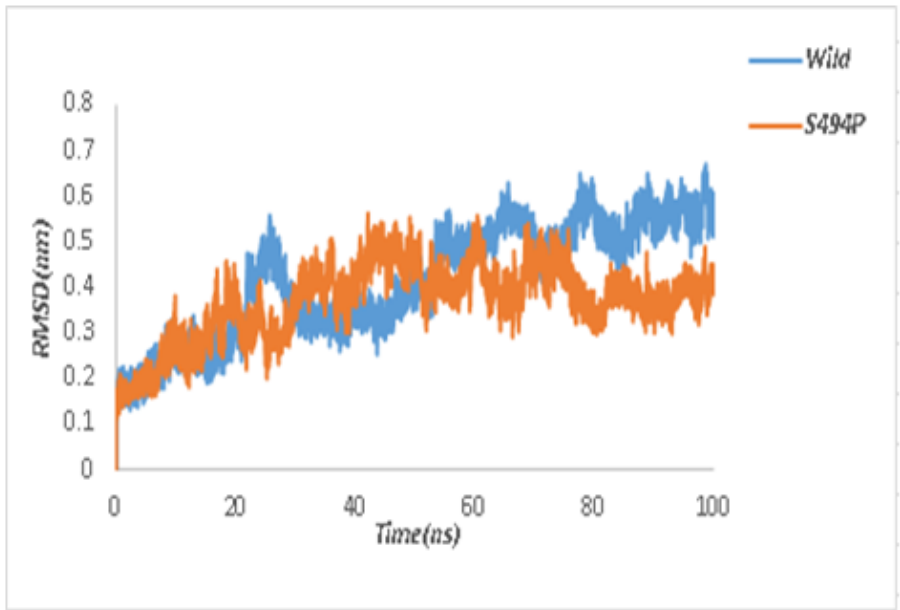

B

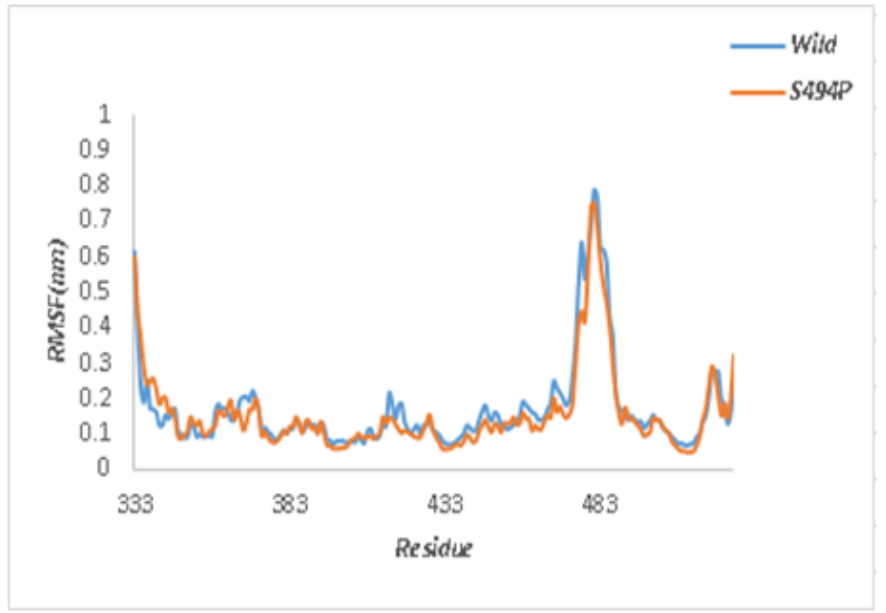

C

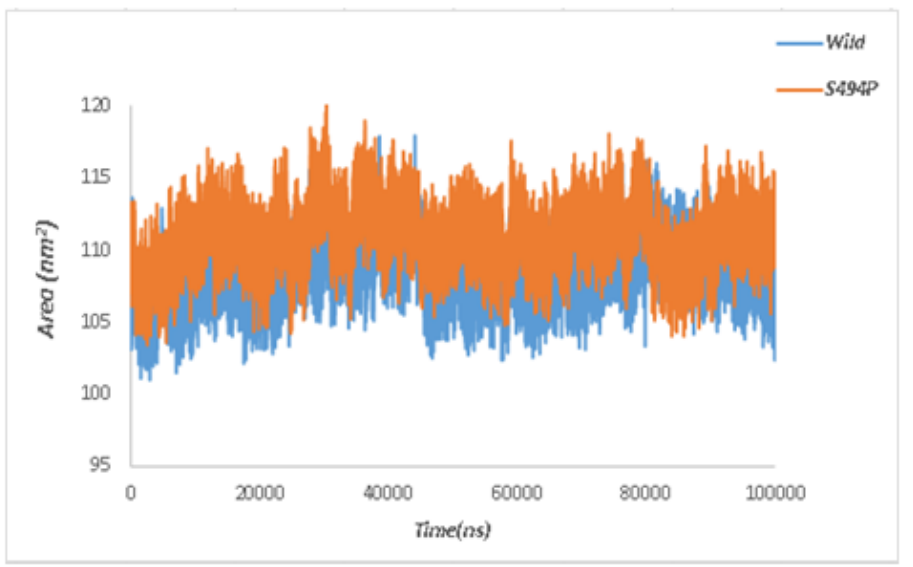

Figure 2

A, Root-mean square deviation (RMSD); B, Root-mean-squared fluctuation (RMSF); and C, the Solvent accessible surface area (SASA)during MD simulation of native and mutant structures.

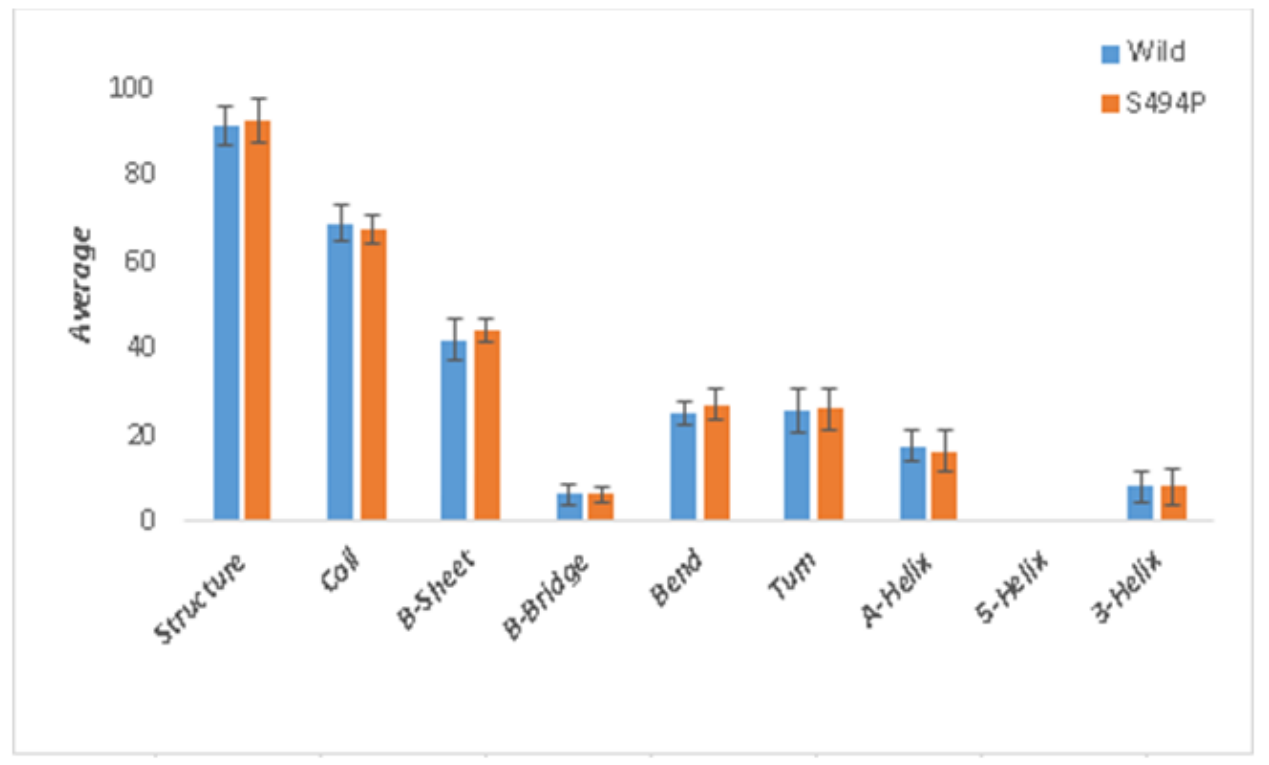

\section{Figure 3}

Average of secondary structures during the MD simulation of wild type and mutant spike proteins. Structure=A-Helix+B-Sheet+B-Bridge+Turn. 
WT

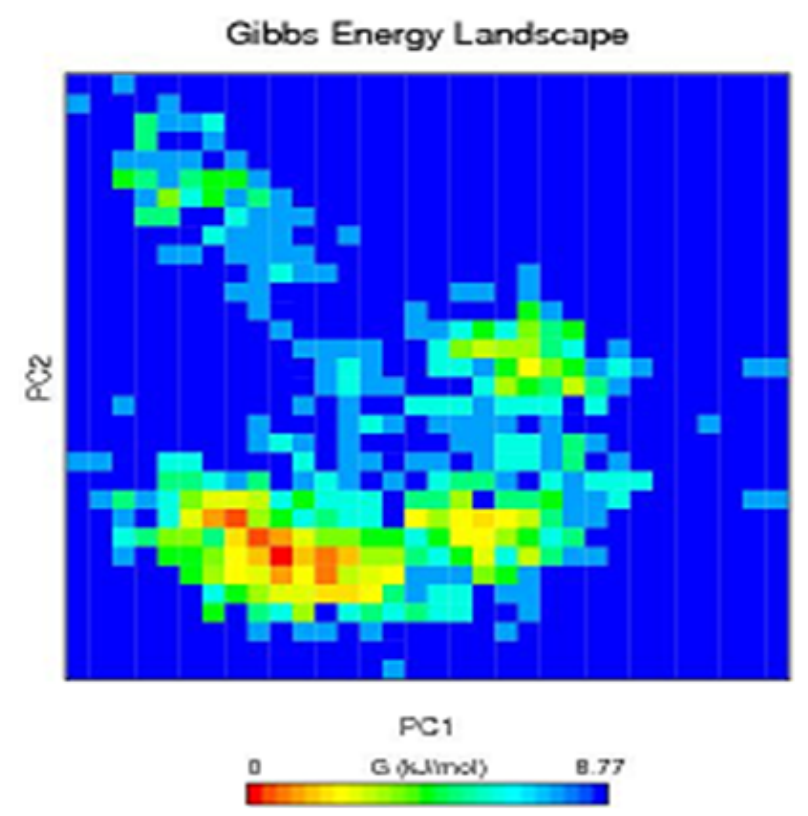

P494

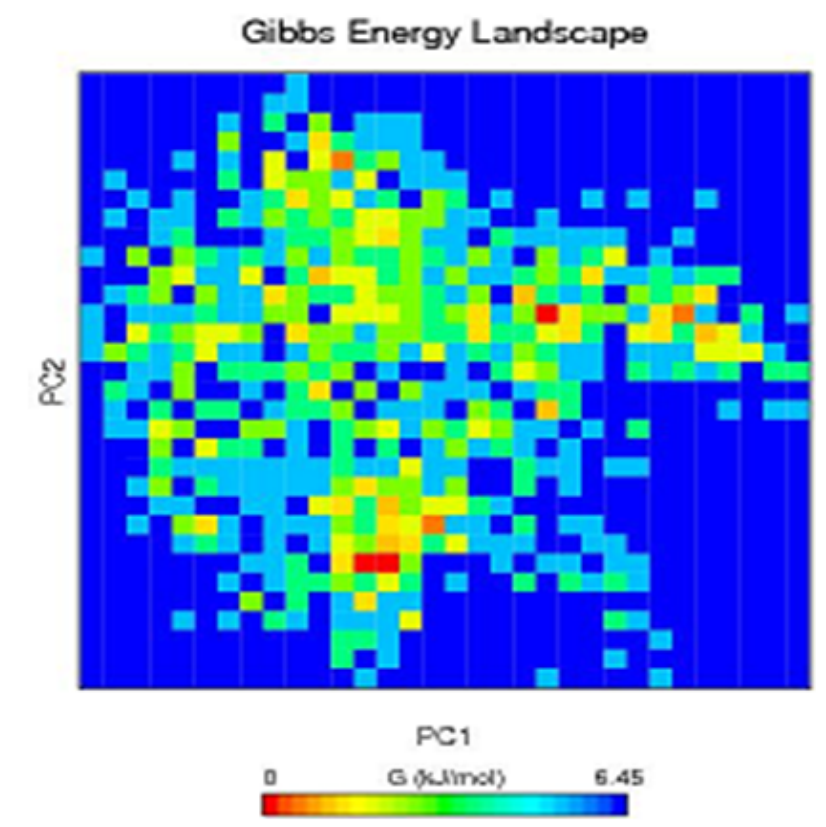

\section{Figure 4}

Projections of Free energy landscape of wild and mutant conformational space onto PC1 and PC2 produced from PCA of Desmond trajectories. The free energy landscape along the first two principal components PC1 and PC2 for (A) wild type and (B) S494P where red and yellow indicates energy minima configuration and blue shows the highest energy configuration. 

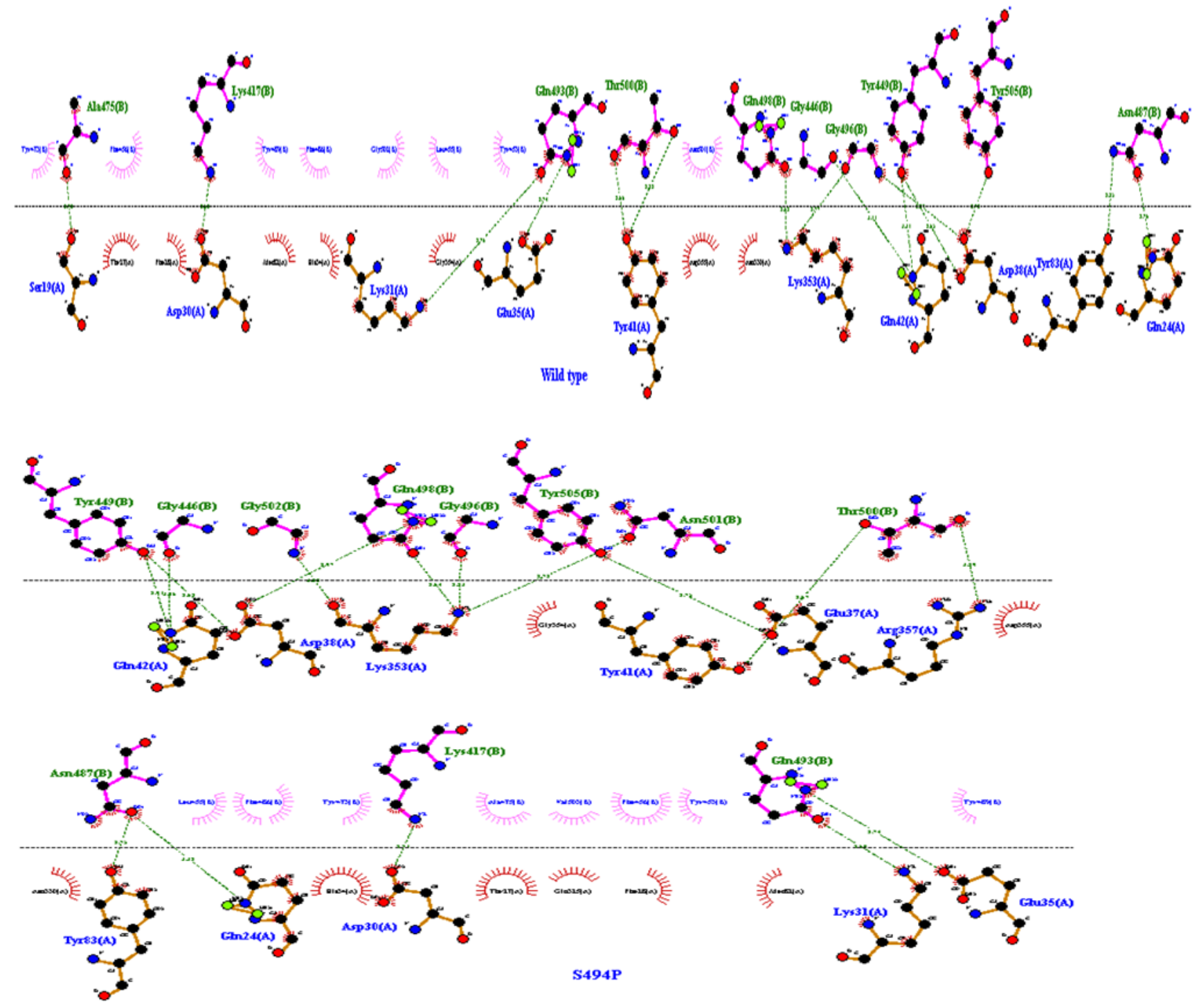

Figure 5

Graphical representation of docking results. Figure panels from up to down represent the 2D interactions of Wild type, and P494 Spikes with hACE2, respectively. 
S494

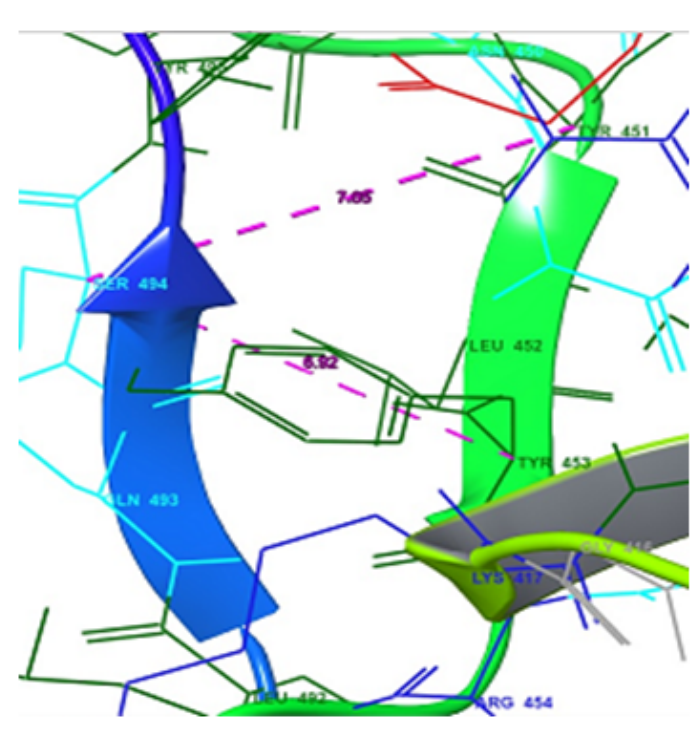

P494

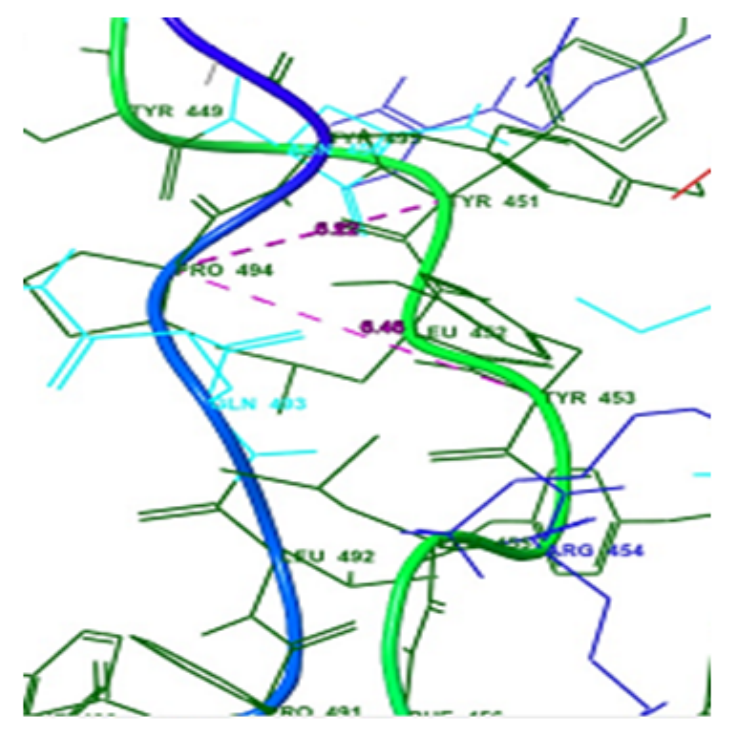

Figure 6

Displays the distance change between position 494 (S494 and P494) relative to other adjacent positions of the structures. Also, conversion of beta structure to loop is seen in P494. 